\title{
Análisis crítico de un artículo: El tratamiento hipoglicemiante intensivo ¿reduce los eventos cardiovasculares 0 la mortalidad en diabéticos tipo 2?
}

\author{
Solange Rivera $M^{1,2}$, Reinaldo U ribe-San Martín ${ }^{3 a}$, \\ Nicolás Belmar N ${ }^{3 a}$, Alfonso Pérez I ${ }^{3 a}$. \\ Critically appraised article: \\ Effects of intensive glucose lowering \\ in type 2 diabetes \\ $N$ Engl J Med 2008; 358: 2545-59. \\ Action to Control Cardiovascular Risk in \\ Diabetes Study Group Gerstein HC, \\ Miller ME, Byington RP, Goff DC Jr, Bigger JT, \\ Buse JB, Cushman WC, Genuth $S$, \\ Ismail-Beigi F, Grimm RH Jr, Probstfield JL, \\ Simons-Morton DG, Friedewald WT.
}

\begin{abstract}
Epidemiologic studies have shown a relationship between glycated hemoglobin levels and cardiovascular events in patients with type 2 diabetes. We investigated whether intensive therapy to target normal glycated hemoglobin levels would reduce cardiovascular events in patients with type 2 diabetes who had either established cardiovascular disease or additional cardiovascular risk factors. Methods: In this randomized study, 10,251 patients (mean age, 62.2 years) with a median glycated hemoglobin level of $8.1 \%$ were assigned to receive intensive therapy (targeting a glycated hemoglobin level below 6.0\%) or standard therapy (targeting a level from 7.0 to 7.9\%). Of these patients, 38\% were women, and 35\% had had a previous cardiovascular event. The primary outcome was a composite of nonfatal myocardial infarction, nonfatal stroke, or death from cardiovascular causes. The finding of higher mortality in the intensive-therapy group led to a discontinuation of intensive therapy after a mean of 3.5 years of follow-up. Results: At 1 year, stable median glycated hemoglobin levels of $6.4 \%$ and $7.5 \%$ were achieved in the intensivetherapy group and the standard-therapy group, respectively. During follow-up, the primary outcome occurred in 352 patients in the intensive therapy group, as compared with 371 in the standard-therapy group (hazard ratio, 0.90; 95\% confidence interval [CI] 0.78 to $1.04 ; \mathrm{P}=0.16$ ). At the same time, 257 patients in the intensive-therapy group died, as compared with 203 patients in the standard-therapy group (hazard ratio, $1.22 ; 95 \% \mathrm{CI}, 1.01$ to $1.46 ; \mathrm{P}=0.04)$. Hypoglycemia requiring assistance and weight gain of more than $10 \mathrm{~kg}$ were more frequent in the intensive-therapy group $(\mathrm{P}<0.001)$. Conclusions: As compared with standard therapy, the use of intensive therapy to target normal glycated hemoglobin levels for 3.5 years increased mortality and did not significantly reduce major cardiovascular events. These findings identify a previously unrecognized harm of intensive glucose lowering in high-risk patients with type 2 diabetes. (ClinicalTrials.gov number, NCT00000620.).
\end{abstract}

\footnotetext{
${ }^{1}$ Unidad de Medicina Basada en Evidencia, Pontificia Universidad Católica de Chile.

${ }^{2}$ Departamento de Medicina Familiar, Pontificia Universidad Católica de Chile.

${ }^{3}$ Escuela de Medicina, Pontificia Universidad Católica de Chile. Santiago de Chile.

aAlumno de Medicina
}

Correspondencia a: Dra. Solange Rivera. E mail: strivera@uc.cl 


\section{CONCLUSIÓN DE LOS REVISORES:}

La terapia hipoglicemiante intensiva en pacientes diabéticos tipo 2, no reduce los eventos cardiovasculares mayores e incrementa la mortalidad a los 3,5 años de seguimiento.

\section{PREgUNTA CLÍNICA:}

¿En diabéticos tipo 2, la terapia hipoglicemiante intensiva, comparada con tratamiento estándar, reduce los eventos cardiovasculares mayores y la mortalidad?

\section{ConTeXto:}

La diabetes mellitus (DM) continúa aumentando su prevalencia ${ }^{1}$. El control de los factores de riesgo cardiovascular sería importante en la prevención de complicaciones macrovasculares, ya que las microvasculares se asocian con el control de la glicemia ${ }^{2}$. Estudios epidemiológicos muestran que un aumento de 1\% de la hemoglobina glicosilada (HbA1c) se asocia con un aumento de $18 \%$ del riesgo de eventos cardiovasculares ${ }^{3}$, un aumento de $12 \%$ a $14 \%$ del riesgo de muerte 2,4 y un aumento de $37 \%$ de riesgo de retinopatía 0 insuficiencia renal ${ }^{2}$. Esto generó la hipótesis de que reducir los valores de HbA1c tendría beneficios. El objetivo del estudio ACCORD fue evaluar el impacto de 3 intervenciones en mortalidad y eventos cardiovasculares de pacientes diabéticos tipo 2 , siendo analizada en este artículo, la publicación correspondiente al punto 1:

1. Control intensivo de glicemia, logrando HbA1c $<6 \%$.

2. Uso de fibratos y estatinas para controlar nivel de colesterol HDL, triglicéridos y colestrol LDL v/s estatinas aisladas (en pacientes con adecuado control glicémico).

3. Control intensivo de la HTA logrando presiones sistólicas menores a $120 \mathrm{mmHg}$ vs menores a $140 \mathrm{mmHg}$ (en pacientes con adecuado control glicémico).

\section{CARACTERÍSTICAS DEL ESTUDIO:}

Tipo de estudio: Estudio clínico aleatorizado, multicéntrico. Pacientes: 10.251 pacientes con DM tipo 2, edad promedio de 62,2 años ( \pm 6,8 años), con antecedentes de enfermedades cardiovasculares o evidencias anatómicas de ateroesclerosis, albuminuria, hipertrofia ventricular izquierda o >2 factores de riesgo cardiovascular (dislipidemias, hipertensión arterial, tabaquismo, obesidad). Se incluyeron pacientes con hemoglobina glicosilada (HbA1c) de $7,5 \%$ o más y el promedio de los grupos fue de 8,1. Se excluyeron pacientes con antecedentes de hipoglicemias frecuentes, incapacidad de mantener monitoreo o uso de insulina en domicilio, IMC $>45 \mathrm{y}$ creatinina $>1,5 \mathrm{mg} / \mathrm{dl}$. Intervención: 5.128 pacientes sometidos a tratamiento intensivo: uso de hipoglicemiantes orales e insulina necesarios para lograr una HbA1c objetivo $<6 \%$ (a criterio del tratante y según paciente: metformina, repaglinida, glimepiride, thiazolidinedionas, inhibidor alfa-glucosidasa, insulina) y un mayor número de controles médicos. Comparación: 5.123 pacientes con tratamiento estándar, cuyo objetivo fue tener HbA1c entre 7\% y 7,9\%. Outcomes: primario: Eventos cardiovasculares mayores (infarto agudo al miocardio no fatal, accidente cerebrovascular no fatal o muerte por causas cardiovasculares); secundarios: mortalidad general o de causa cardiovascular. Efectos adversos: Hipoglicemias requiriendo asistencia médica, incremento de peso. Seguimiento: planificado de 5,6 años, que fue detenido a los 3,5 años. 
VALDEZ:

Aleatorizado:

Ocultamiento de secuencia de aleatorización:

Sí

Seguimiento:

Detención precoz:

Análisis con intención de tratar:

Fueron ciegos a la intervención

Pacientes:

Tratantes:

Recolectores de datos:

Adjudicadores de eventos:

Análisis de datos:

Grupos similares respecto a variables pronósticas conocidas:
Sí

$98 \%$

Si*

Sí

No

No

No consignado

Sí

No consignado

Sí

$*_{\text {no }}$ por beneficio

Resultados:

\begin{tabular}{|c|c|c|c|c|}
\hline O utcome & $\begin{array}{c}\text { Grupo } \\
\text { intensivo } \\
\text { n (\%) }\end{array}$ & $\begin{array}{l}\text { Grupo } \\
\text { estándar } \\
\text { n (\%) }\end{array}$ & $\begin{array}{c}\text { HR } \\
\text { (IC 95\%) }\end{array}$ & $\begin{array}{l}\text { NNT/N NH } \\
\text { (IC 95\%) }\end{array}$ \\
\hline $\begin{array}{l}\text { Eventos C-V mayores } \\
\text { (outcome compuesto)* }\end{array}$ & $352(6,9)$ & $371(7,2)$ & $\begin{array}{c}0,9 \\
(0,78-1,04)\end{array}$ & NS \\
\hline Mortalidad general & $257(5,0)$ & $203(4,0)$ & $\begin{array}{c}1,22 \\
(1,0-1,5)\end{array}$ & $\begin{array}{c}100 \\
(55-506)\end{array}$ \\
\hline Mortalidad cardiovascular & $135(2,6)$ & $94(1,8)$ & $\begin{array}{c}1,35 \\
(1,0-1,8)\end{array}$ & $\begin{array}{c}125 \\
(73-430)\end{array}$ \\
\hline AVE*** no fatal & $67(1,3)$ & $61(1,2)$ & $\begin{array}{c}1,06 \\
(0,75-1,50)\end{array}$ & NS \\
\hline $\begin{array}{l}\text { Infarto agudo al miocardio } \\
\text { no fatal }\end{array}$ & $186(3,6)$ & $235(4,6)$ & $\begin{array}{c}0,76 \\
(0,62-0,92)\end{array}$ & $\begin{array}{l}100(\mathrm{NNT}) \\
(57-430)\end{array}$ \\
\hline $\begin{array}{l}\text { Hipoglicemia } \\
\text { (asistencia médica) }\end{array}$ & $538(10,5)$ & $179(3,5)$ & $\begin{array}{c}3 \\
(2,6-3,5)\end{array}$ & $\begin{array}{c}14 \\
(13-17)\end{array}$ \\
\hline Aumento de peso $>10 \%$ & $1.399(27,8)$ & $713(14,1)$ & $\begin{array}{c}1,97 \\
(1,8-2,1)\end{array}$ & $\begin{array}{c}7 \\
(7-8)\end{array}$ \\
\hline
\end{tabular}

$\mathrm{HR}=$ Hazard ratio, $\mathrm{NNH}=$ =Número necesario para hacer daño, IC =Intervalo de confianza, NS =No significativo.

* Outcome compuesto "eventos cardiovasculares mayores" incluye mortalidad cardiovascular, AVE no fatal, infarto agudo al miocardio no fatal.

*AVE accidente vascular encefálico

\section{COMENTARIOS Y APLICACIÓN PRÁCTICA:}

- Este estudio cumple la mayoría de los criterios de validez de un estudio clínico randomizado, destacan la imposibilidad de realizar una intervención ciega (ya que el 
grupo intervenido requirió mayor cantidad de hipoglicemiantes, insulina y visitas médicas) y la detención precoz del estudio aunque no por beneficio. El riesgo de sesgo es bajo ya que los outcomes principales fueron objetivos (mortalidad y eventos cardiovasculares) y los adjudicadores de eventos fueron ciegos a la intervención. La detención precoz fue por el daño detectado en los análisis interinos (aumento en la mortalidad general), lo que en términos éticos se condice con el principio de no hacer daño (primum non nocere) que obliga a detener el estudio ${ }^{5}$.

- Los resultados muestran que la terapia hipoglicemiante intensiva en DM tipo 2, no reduce todos los eventos cardiovasculares mayores. Si bien se redujo el riesgo relativo de infarto al miocardio en 24\%; por otra parte se observó un incremento relativo de $22 \%$ en la mortalidad general y de $35 \%$ en la mortalidad cardiovascular a los 3,5 años de seguimiento. Estos resultados fueron ajustados por edad, sexo, raza, eventos cardiovasculares previos, siendo la presencia de una HbA1c $<8 \%$ al inicio del estudio, el único factor asociado a una mayor tasa de eventos cardiovasculares.

- El outcome primario corresponde a un outcome compuesto (concepto que se ha profundizado en el presente número de la Revista Médica de Chile). Si bien este outcome compuesto (infarto agudo al miocardio no fatal, accidente cerebrovascular no fatal y mortalidad cardiovascular) es en teoría razonable, la principal dificultad que tendría utilizarlo en la toma de decisiones clínicas es que el efecto sobre los distintos componentes fue completamente diferente (beneficio sobre el infarto, efecto neutro sobre el accidente vascular y aumento de la mortalidad).

- Dentro de las posibles causas que podrían explicar la mayor mortalidad cardiovascular observada en la terapia intensiva, cabe destacar el mayor uso de tiazolidinedionas respecto al tratamiento estándar, 91,7\% versus 58,3\%, respectivamente. Estos fármacos han sido asociados posteriormente con mayor riesgo de eventos cardiovasculares ${ }^{6,7}$.

- El grupo intervenido presentó mayor porcentaje de hipoglicemias que requirieron de asistencia médica $(10,5 \%$ vs $3,5 \%$ del grupo estándar). Además, la terapia intensiva presentó una tasa de efectos adversos distintos de hipoglicemia de 2,2\% vs 1,6\% en el grupo control.

- Paralelamente al estudio ACCORD, se desarrolló el estudio ADVANCE (Action in Diabetes and Vascular Disease: Preterax and Diamicron Modified Release Controlled Evaluation $)^{8}$ que buscaba determinar los potenciales beneficios de tratar en forma intensiva (HbA1c $<6,5 \%$ ) a pacientes con DM tipo 2. La población del estudio y el tiempo de seguimiento eran similares a las del ACCORD, observándose en el grupo intensivo, una reducción de los eventos microvasculares. Respecto a los eventos macrovasculares existe una tendencia a la disminución con el tratamiento intensivo, después de los 5 años de seguimiento, sin embargo, estas diferencias no fueron significativas. Sería interesante analizar en ambos estudios si existe una correlación entre el nivel de hemoglobina glicosilada y mortalidad, para así identificar el nivel de menor riesgo para estos pacientes.

- Considerando los resultados de ambos estudios, en los pacientes diabéticos tipo 2 en tratamiento intensivo, existiría un beneficio al reducir las complicaciones microvasculares, pero no está claro su impacto en mortalidad y eventos cardiovasculares.

- Los pacientes del estudio fueron similares a nuestra población: edad promedio cercana a 60 años, 10 años de duración de diabetes, 35\% con eventos cardiovasculares previos. Sin embargo, sólo 7\% del estudio era de origen hispánico.

- Es importante destacar que las metas propuestas por el Ministerio de Salud de Chile para el control glicémico en pacientes diabéticos, tienen como objetivo una hemoglobina glicosilada $<7 \% 9$, pudiendo según lo observado en este estudio, aumentar la mortalidad en aquellos pacientes en control glicémico más estricto. 
- La Asociación Americana de Diabetes (ADA) presentó una declaración sobre este artículo, esperando con interés un análisis más detallado de los datos, así como otros estudios en curso que pueden arrojar más luz sobre estos hallazgos. Actualmente, la ADA aconseja a las personas con DM que tienen enfermedades cardiovasculares 0 múltiples factores de riesgo, consultar con su equipo de atención médica acerca de sus objetivos del tratamiento y asegurar que su presión arterial y el colesterol sean manejados adecuadamente ${ }^{10}$.

- En resumen, el tratamiento intensivo de la DM tipo 2, definido en este estudio como la obtención de HbA1c menor a $6 \%$, es una alternativa terapéutica que aún debe ser sustentada por nuevos estudios clínicos randomizados, tratando de establecer un balance entre los ya conocidos beneficios microvasculares y el riesgo de aumentar la tasa de eventos cardiovasculares detectados en este estudio.

\section{REFERENCIAS}

1. Encuesta Nacional de Salud Chile 2003. http://epi.minsal.cl/html/invest/ENS/ENS.htm.

2. Stratton im, Adier AI, Neil HA, Matthews DR, Manley SE, Cull CA et al. Association of glycaemia with macrovascular and microvascular complications of type 2 diabetes (UKPDS 35): prospective observational study. BMJ 2000; 321: 405-12.

3. Selvin E, Marinopoulos S, Berkenblt G, Rami T, Brancati FL, Powe NR et al. Meta-analysis: glycosylated hemoglobin and cardiovascular disease in diabetes mellitus. Ann Intern Med 2004; 141: 421-31.

4. Gerstein HC, Pogue J, Mann JF, Lonn E, Dagenai GR, McQueen M, Yusuf S; Hope Investigators. The relationship between dysglycaemia and cardiovascular and renal risk in diabetic and non-diabetic participants in the HOPE study: a prospective epidemiological analysis. Diabetologia 2005; 48: $1749-55$.

5. CANDia R, Letelier LM, RADA G. Estudios randomizados detenidos precozmente por beneficio: ¿Muy buenos o muy malos? Rev Méd Chile 2006; 134: 1470-5.

6. Lago RM, Singh PP, NeSto RW. Congestive heart failure and cardiovascular death in patients with prediabetes and type 2 diabetes given thiazolidinediones: a meta-analysis of randomised clinical trials. Lancet 2007; 370: 1129-36.

7. Eurich DT, Mcalster Fa, Blackburn DF, Majumdar SR, Tsuyuki RT, Varney J, Johnson Ja. Benefits and harms of antidiabetic agents in patients with diabetes and heart failure: systematic review. BMJ 2007; 335: 497-506.

8. Advance Colaborative Group. Patel A, MacMahon S, Chalmers J, Neal B, Billot L, Woodward M, Marre $\mathrm{M}$ et al. Intensive Blood Glucose Control and Vascular Outcomes in Patients with Type 2 Diabetes. N Engl J Med 2008; 358: 2560-72.

9. Ministerio de SAlud. Guía Clínica: Diabetes Miellitus tipo 2. Serie Guías Clínicas MINSAL № XX, 1a edición. Santiago: Minsal, 2006.

10. Statement from the American Diabetes Association related to the ACCORD trial Announcement. http:// www.diabetes.org/formedia/pr-ada-statement-related-to-accord-trail-announcement020608.jsp. 